\title{
A new and updated resource for codon usage tables
}

\author{
John Athey ${ }^{1 \dagger}$, Aikaterini Alexaki ${ }^{1 \dagger}$, Ekaterina Osipova ${ }^{2}$, Alexandre Rostovtsev ${ }^{2}$, Luis V. Santana-Quintero ${ }^{2}$, \\ Upendra Katneni ${ }^{1}$, Vahan Simonyan ${ }^{2}$ and Chava Kimchi-Sarfaty ${ }^{1 *}$ (i)
}

\begin{abstract}
Background: Due to the degeneracy of the genetic code, most amino acids can be encoded by multiple synonymous codons. Synonymous codons naturally occur with different frequencies in different organisms. The choice of codons may affect protein expression, structure, and function. Recombinant gene technologies commonly take advantage of the former effect by implementing a technique termed codon optimization, in which codons are replaced with synonymous ones in order to increase protein expression. This technique relies on the accurate knowledge of codon usage frequencies. Accurately quantifying codon usage bias for different organisms is useful not only for codon optimization, but also for evolutionary and translation studies: phylogenetic relations of organisms, and host-pathogen co-evolution relationships, may be explored through their codon usage similarities. Furthermore, codon usage has been shown to affect protein structure and function through interfering with translation kinetics, and cotranslational protein folding.
\end{abstract}

Results: Despite the obvious need for accurate codon usage tables, currently available resources are either limited in scope, encompassing only organisms from specific domains of life, or greatly outdated. Taking advantage of the exponential growth of GenBank and the creation of NCBI's RefSeq database, we have developed a new database, the High-performance Integrated Virtual Environment-Codon Usage Tables (HIVE-CUTs), to present and analyse codon usage tables for every organism with publicly available sequencing data. Compared to existing databases, this new database is more comprehensive, addresses concerns that limited the accuracy of earlier databases, and provides several new functionalities, such as the ability to view and compare codon usage between individual organisms and across taxonomical clades, through graphical representation or through commonly used indices. In addition, it is being routinely updated to keep up with the continuous flow of new data in GenBank and RefSeq.

Conclusion: Given the impact of codon usage bias on recombinant gene technologies, this database will facilitate effective development and review of recombinant drug products and will be instrumental in a wide area of biological research. The database is available at hive.biochemistry.gwu.edu/review/codon.

Keywords: Codon usage bias, Codon optimization, Recombinant protein therapeutics, Translational kinetics

\section{Background}

There are 64 possible nucleotide triplet combinations but only 20 amino acids to encode; as a result, most amino acids can be encoded by more than one codon. Codons that are translated to the same amino acid are called synonymous. In each organism there is a

\footnotetext{
* Correspondence: Chava.Kimchi-Sarfaty@fda.hhs.gov

${ }^{\dagger}$ Equal contributors

'Division of Plasma Protein Therapeutics, Office of Tissue and Advanced Therapies, Center for Biologics Evaluation and Research, Food and Drug Administration, Silver Spring, USA

Full list of author information is available at the end of the article
}

preference for certain codons over others; therefore, synonymous codons occur with different frequencies, a phenomenon termed codon usage bias, which is observed across species, albeit with different intensities [1]. Two major hypotheses have been proposed for explaining the existence of codon usage bias. One argues that codon usage bias contributes to the efficiency and accuracy of protein translation and is therefore maintained by selection [2]. The other claims that codon usage bias exists because of the non-randomness in mutational patterns, whereby some codons may be more prone to mutation than others and are therefore 
found at different frequencies [3]. These hypotheses are not mutually exclusive [4-7]. Relative synonymous codon usage (RSCU), Codon adaptation index (CAI) [8], effective number of codons (ENc) [9] and tRNA adaptation index (tAI) [10] are commonly used metrics, aiming to quantitate codon bias in a gene or a species.

We have generated a database, the HIVE-Codon Usage Tables (HIVE-CUTs), presenting the codon usage statistics for every organism that has available compiled sequencing data. The sequence data collected for this analysis have been derived from the GenBank [11] and RefSeq [12] databases. The codon usage tables are linked to a taxonomy tree to allow comparative analysis of the codon usage frequencies. Knowing the frequency of occurrence of codons within a genome is essential in common biological techniques and in a number of fields of study. Codon optimization, which involves replacing rare codons with frequent ones, requires knowledge of the preferred codons in a given organism. Furthermore, synonymous codon usage patterns can be an essential tool in revealing evolutionary relationships between species as well as host-pathogen coevolution and adaptation of pathogens to specific hosts [13-17]. Interestingly, some viruses appear to take advantage of the codon usage of their host to temporally regulate late expression of their proteins [18]. An area of research that is currently gaining attention pertains to how codon usage may affect protein structure. It has long been assumed, based on Anfinsen's theorem [19], that since synonymous mutations do not affect the primary structure of a protein, they also should not affect the secondary and tertiary structure. However, recent data have suggested that this assumption is untrue; synonymous codon changes can profoundly affect the translation rate of a protein, which in turn may modulate the folding of the nascent polypeptide chain [20-24]. As the translational kinetics of a protein depend, at least in part, on the frequency of its codons, having access to codon usage information can be valuable in determining effects of synonymous mutations on protein structure. It should be noted that synonymous mutations may have multiple other effects on protein expression and function beyond translational kinetics that may instead be linked to effects on nucleosome structure, transcription factor binding, splicing efficiency, RNA-protein interactions, microRNA binding, and RNA secondary structure [24-28]. These effects of synonymous mutations, although of high importance, are not directly related to codon frequency and therefore will not be further discussed here.

Despite the applicability of codon usage tables to many areas of research, currently available resources provide data that are limited, inaccurate or out of date. Some existing databases contain information on bacterial and archaeal genomes but not on eukarya and viruses
[29, 30]. The widely used Kazusa database, on the other hand, includes information on all domains of life but has not been updated since 2007 [31]. However, following the rapid development of high-throughput sequencing over the last few years, the amount of sequence information available has drastically increased. The last update of the Kazusa database (GenBank release 160, June 2007) contained just over 3 million coding sequences (CDSs); in comparison, this new database analyses 35 million CDSs from GenBank and another 255 million from RefSeq. For many organisms in the Kazusa database, the number of CDSs included was too low to be useful; for example, the codon usage table for the western lowland gorilla (Gorilla gorilla gorilla) was based on only two coding sequences. These shortcomings are widely recognized by researchers who often generate new codon usage tables for the species they are studying [32, 33]. However, this process, in addition to being labour intensive and requiring computational knowledge, may create substantial variability, as the databases from which the sequences are retrieved change over time and different criteria may be applied in the inclusion of sequences for analysis. The presented HIVECUTs database, on the other hand, will be updated every 2 months, corresponding with GenBank releases, and each version of the database will remain available to provide a stable reference.

Furthermore, compared to the Kazusa database the HIVE-CUTs database has the advantage of utilizing both GenBank and RefSeq sequences separately. The incorporation of RefSeq data into the proposed database is a critical development that is necessary to provide researchers the most accurate data available, and warrants the creation and maintenance of this new database. NCBI's RefSeq database aims to minimize redundancy and provide high quality annotations, and provides a data source that was not included in the older database. For example, in the Kazusa database, the sequence for human coagulation factor IX, a single copy gene, was included 13 times due to the inclusion of multiple submissions by various groups. However, the same analysis performed on the RefSeq Homo sapiens assembly would include this gene only twice-the wild type sequence and an alternative splicing variant. Overall, larger and more accurate sources of sequencing data have made the generation of current codon usage tables a necessity for a wide range of applications.

\section{Construction and content Input data}

Codon usage for all available organisms was computed separately for both the GenBank and RefSeq databases at NCBI. Data from GenBank was derived from GenBank release 215.0 (released August 152016 [11]), while 
RefSeq data [12] used all assemblies that were current as of September 29 2016. Within the RefSeq division, all genome assemblies that were designated "latest" are included in the database. These assemblies were selected by parsing the RefSeq "assembly summary" files available on NCBI. For GenBank, the divisions incorporated into our codon usage database are BCT (bacterial), PRI (primate), ROD (rodent), MAM (other mammalian), VRT (other vertebrate), INV (invertebrate), PLN (plant and fungal), VRL (viral), and PHG (phage). The other divisions published by GenBank do not derive from organismal sources (e.g. the "EST" (expressed sequence tag) division), or come from organisms with no assigned names (e.g. "ENV" (environmental samples)). In total, 288 million coding sequences (35 million from GenBank, 253 million from RefSeq) were included in the database, resulting in the creation of over 855,000 codon usage tables.

\section{Data processing}

The data from both divisions was processed using Python 2.7, using the Biopython module (version 1.68) to parse the annotated genome features [34]. Each record was processed according to the tags available in the file; only protein coding sequences ("CDS" tag) were included for codon usage and GC percent analysis. Pseudogenes and "low quality" proteins (transcripts with a corrected base relative to the genome) were excluded from the analysis. The taxID number of the organism is parsed from the "db_xref" tag in the file, while the scientific name of the organism is retrieved from NCBI's taxonomy database [35]. Features for which the annotated sequence could not be extracted were also excluded; as a result, records with unusual tags may not have been included, and records that specified their sequence data via another accession record were not included. However, the number of records excluded is low and should not affect the quality of the data overall. Features containing ambiguous nucleotides were included, but the individual codons containing ambiguous nucleotides were excluded. Other information that is parsed from each record includes the translation table and accession number of each individual CDS. The actual execution of data download and parsing was performed using High-performance Integrated Virtual Environment (HIVE) platform [36]. HIVE was originally created and optimized for loading, parsing, storage, and analysis of extra-large datasets.

\section{Output and organization}

The resulting codon usage tables are organized in dictionaries by assembly accession numbers for RefSeq, or by the species name for GenBank. This means that multiple genome submissions for a given organism are combined in GenBank, but are separate entries in RefSeq. Additionally, mitochondrial, chloroplast, plastid, leucoplast, and chromoplast genes are considered separate from the genomic data, and have their own organellar codon usage tables. However, as plasmids are located in the same area as the genome, draw from the same tRNA pools, and use the same genetic code as the genome, plasmid coding sequences are not separated from the organism's genomic codon usage table. The resulting codon usage tables can be downloaded as a tabular text file, or searched through using our publicly accessible web interface. To obtain the data that best represents the codon usage of an organism, users should search for a single RefSeq assembly, as this is derived from a single sequence assembly from a single organism. GenBank data is compiled from any number of different submissions of varying completion status, and may therefore be skewed when searching for a single organism (see Additional file 1). However, GenBank contains much more data deriving from many more organisms than RefSeq, so even though it may not provide the most accurate data for an organism's codon usage, it will be of use for less well studied organisms. Additionally, codon usage tables for each CDS, as opposed to each organism, are also produced by the program; these tables cannot currently be viewed via the web, but can be downloaded and parsed. The files available for download through the website are tabular text files comprised of codon usage tables organized either by species/assembly or coding sequence; each entry contains information about the record (e.g. assembly number and DNA type) and the totals for each codon. Table 1 indicates the magnitude of the database, including the number of tables and species included, as well as the distribution of tables between GenBank and RefSeq and between genomic and other organellar tables. Furthermore, each version of the database will be accessible through a stable identifier, allowing researchers to always reference a consistent version of the database.

Table 1 HIVE-CUT database size and statistics

\begin{tabular}{llll}
\hline Measure & GenBank & RefSeq & Total \\
\hline Number of tables & 781,595 & 73,817 & 855,412 \\
Number of species & 665,044 & 37,904 & 689,420 \\
Genomic tables & 353,423 & 73,553 & 426,976 \\
Mitochondrial tables & 316,820 & 220 & 317,040 \\
All plastid tables & 111,352 & 44 & 111,396 \\
Total number of sequences & $34,885,329$ & $253,803,831$ & $288,689,160$
\end{tabular}

This table contains statistics on the data in the database. While the GenBank division contains a much larger number of tables, the number of sequences in each table on average is much higher in RefSeq. The structure of RefSeq assemblies makes them a better representation of genomic codon usage for an organism when available. The HIVE-CUTs database contains substantially more entries than other codon usage databases 
The ENc has been adapted from Wright 1990 [9]. Instead of being calculated on a per gene basis, one ENc value is computed for the entirety of the genomic coding sequences. In addition, stop codons are also considered and included in the calculation as any other amino acid. The ENc is also calculated using each different genetic code, and users can select the one most appropriate for their organism of interest.

\section{Website interface}

HIVE is used to implement the data-storage and visualization web-portal for this project. Its interface follows Data-Driven Documents (DDD) paradigm instead of static HTML pages to tackle the visualization challenges presented by the outputs from large data sets [37]. The separation among content, functionality, and object model is the underlying concept of HIVE's interface achieved by construction of Document Object Model (DOM) model on the client side. This communication is done asynchronously, using an Ajax (Asynchronous Javascript and XML) web application model.

Internally, HIVE is a computer cluster executing a large number of heavily parallelized scientific processes. The front end (user interface) is a simplified representation of an advanced infrastructure that exists on the back end. There are intermediate layers based on
Common Gateway Interface (CGI) and SQL database that help with the communication between front end and back end. As the user waits for the service to be completed, a daemon process is responsible for the execution of services, for monitoring progress, and updating the state during the parallel execution. After completion the whole page gets updated with the requested data so the client's browser can launch specific visualization engines. This allows generation of appropriate interactive visualization. In Fig. 1 there is a general workflow of the HIVE platform.

One of the visualization tools that are used on the page is a dynamic Taxonomy Tree that is constructed using the d3js JavaScript library [37]. The information for this tree is derived from taxonomy data held at NCBI (released May 52016 [35]). HIVE's visualization tree is capable of loading information about a specific node including its taxonomy ID, parent, and children information. The other visualization tool being used is Google Charts that are integrated into HIVE's visual library. Google Charts are customizable on many parameters, and these parameters are used to generate a bar chart that allows the users to make a side by side comparison of selected codon tables.

Users can access additional webpages and resources that utilize codon usage tables through the "Other Resources" tab on the webpage.

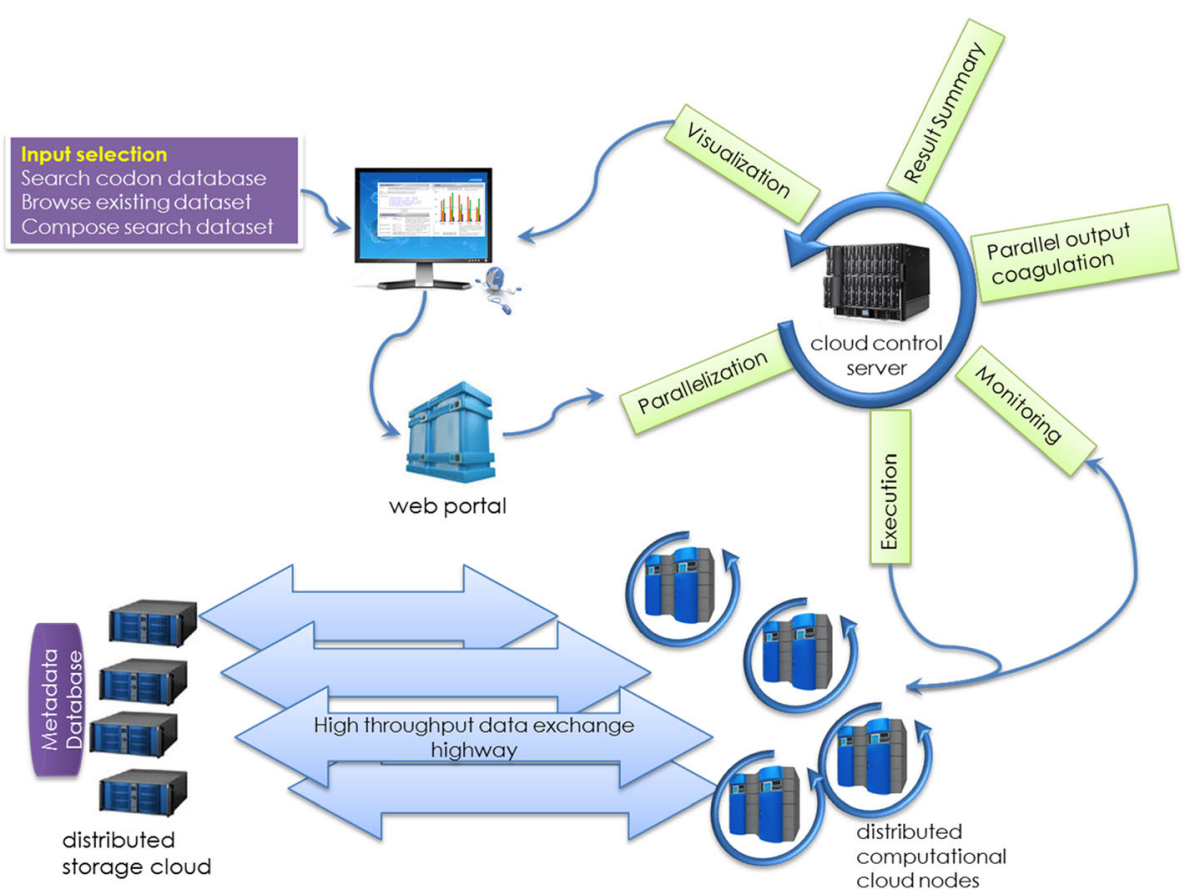

Fig. 1 HIVE Platform [36]. A client process submits the information request from the HTML form or web application into the HIVE server; this request is queued for execution and it is computed inside the distributed environment. The front end monitors the status of the request and once the computation is finished, data is retrieved and visualizations are prepared to be sent to the client's web page 


\section{Utility and discussion}

We have generated new codon usage tables for every organism in GenBank and RefSeq and created a userfriendly platform where codon usage data can be retrieved from a publically available website [38]. The initial HIVE-CUTs webpage contains a search tab, results tab, and help tabs on the right and bottom. The web interface for searching the database features several options for searching the data. Users must decide whether to search through GenBank or RefSeq, as well as what type of data (genomic or another organelle) they want to analyse. Users can search for entries based on scientific names, taxonomical ID numbers (taxID), or (in RefSeq only) assembly accessions. All of these options, except searching for a single assembly, can be applied to any taxonomical rank, allowing users to retrieve and compare data from different clades. When searching for a species-rank scientific name or taxID number, users also have the option to combine entries belonging to sub-species of that entry, by choosing the "deep search" option, e.g. retrieving $E$. coli and all its strains, or only retrieving submissions for $E$. coli with no strain information assigned. Once a search is submitted the results appear in several tables and graphs. Each window and graph can be enlarged or closed. The codon usage tables are in plain text format with each codon, its frequency per 1000 codons, and the raw total for that codon in the genome, in the default order specified by NCBI's standard genetic code definition (Fig. 2). This is a common format and the table can be copied and directly pasted in a number of applications such as ATGme [39] and Rare Codon Calculator: \%MinMax [40]; several such tools are linked directly from the database webpage. Each search will produce two graphs, one plotting the GC percent frequency of the organism's coding sequences as well as at each codon position, and the other plotting the frequencies of each codon per 1000 codons. The graphical presentation of the frequencies of each codon (Fig. 2) can be especially useful when comparing frequencies across different codon usage tables. To enable comparisons between different organisms and clades, multiple queries can be submitted simultaneously; the codon and GC frequencies for each query are plotted both individually and together (Fig. 3). In addition, a text table listing the ENc for each query is generated. ENc is a metric that measures codon bias in terms of deviation from an assumed neutral distribution of synonymous codon usage. Larger ENc values correspond to more equal usage of synonymous codons, while the lowest possible ENc value would result from the case of one codon used for each amino acid [9]. The ENc was calculated for all genomic coding sequences collectively. RefSeq and GenBank do not always assign a genetic code to each genome, therefore, ENc was calculated using each genetic code; users may select the ENc that is appropriate for their organisms of interest. To facilitate studying the evolution of codon usage bias across species we incorporated a visual representation of their taxonomical relationship in our

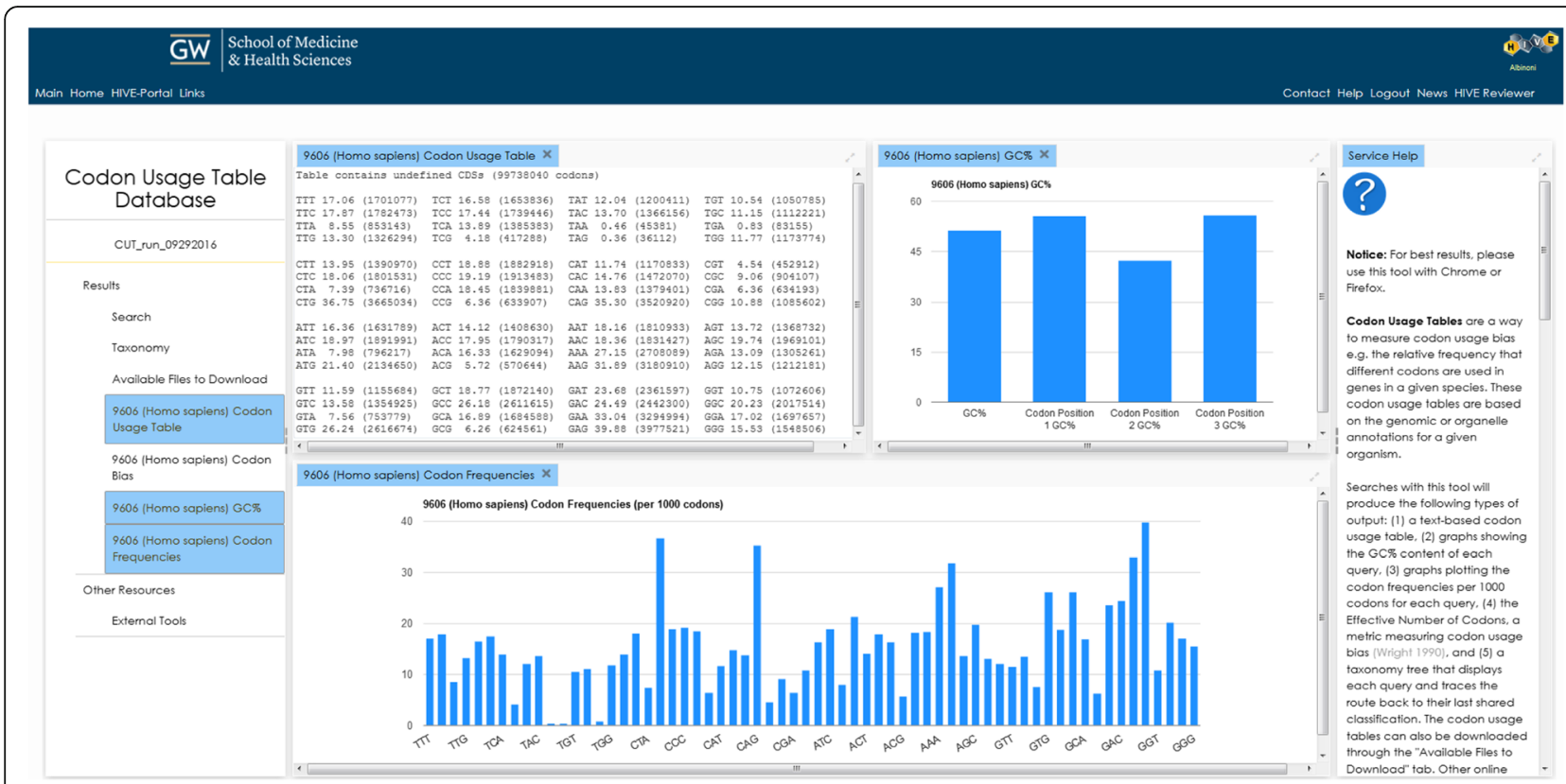

Fig. 2 Screenshot of HIVE-CUTs webpage with Homo sapiens results. Results include codon usage frequencies per 1000 codons as a plain text table (top left) and graph (bottom), in the default order specified by NCBI's standard genetic code definition. The GC frequency in the genome and at each codon position is also presented in a graph (top right). The help panel is included (right) 


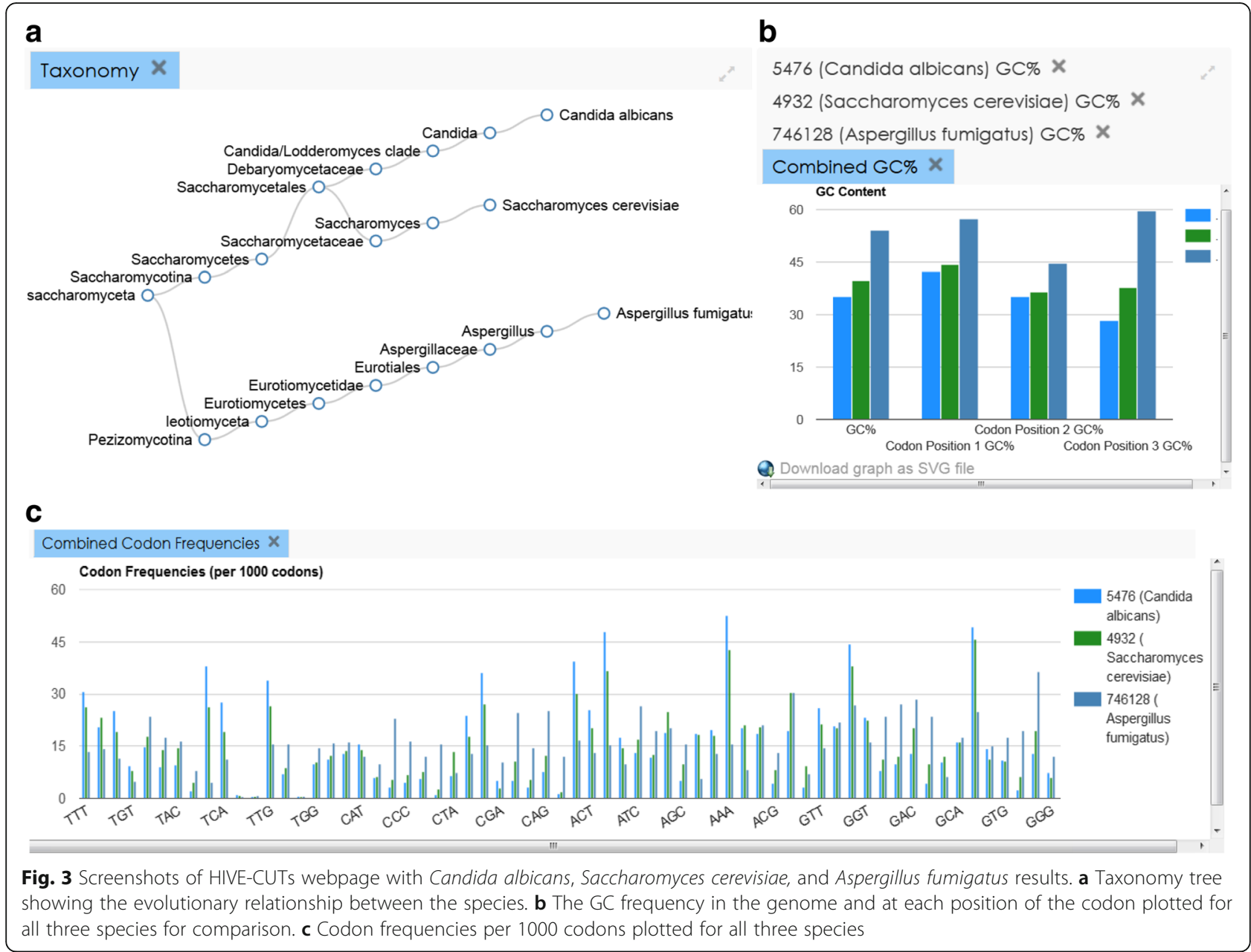

application. When users submit a search for one or more taxonomical nodes, the nodes will be highlighted and the branch will be expanded to show the relationship between them, going back to the highest common classification. Though the tree is taxonomical, not phylogenetic, being able to visualize the distance between organisms of interest and in parallel examine their similarities in terms of codon usage bias can be an instrumental tool in evolutionary studies. In the example shown, Candida albicans and Saccharomyces cerevisiae, two taxonomically close yeast species, are shown along with Aspergillus fumingatus, which is more distantly related. The distance in the taxonomy tree is reflected in the differences of their codon usage tables (Fig. 3).

The HIVE-CUTs may be instrumental in recombinant gene applications such as gene therapy, vaccine development and protein therapeutics, and in a wide area of research including evolution, comparative molecular biology and translation kinetics.

Knowledge of codon usage across species is crucial when recombinant proteins are expressed in heterologous organisms [41-44]. There are several approaches that are commonly used to increase expression of heterologous proteins, including codon optimization [45-47], codon harmonization [48, 49], and supplementation of rare tRNAs [50-53]. When codon usage in the organism of origin is starkly different from that of the organism used for expression, for example when a human gene is expressed in E. coli, codon optimization (i.e. replacement of rare codons with more frequent synonymous codons) can to an increase in expression of a few orders of magnitude $[41,45,54,55]$. Codon harmonization is conceptually similar to codon optimization, wherein codons of the native protein are replaced with synonymous codons that have a similar usage frequency in the heterologous expression host $[48,49]$. However, accurate codon usage data is important for ensuring that optimization or harmonization strategies actually lead to improved expression; if the codon usage data is incomplete or inaccurate, optimization steps could be unsuccessful at increasing expression or may even reduce it. For example, the biotechnology industry often uses Chinese hamster ovary $(\mathrm{CHO})$ cells to express human recombinant 


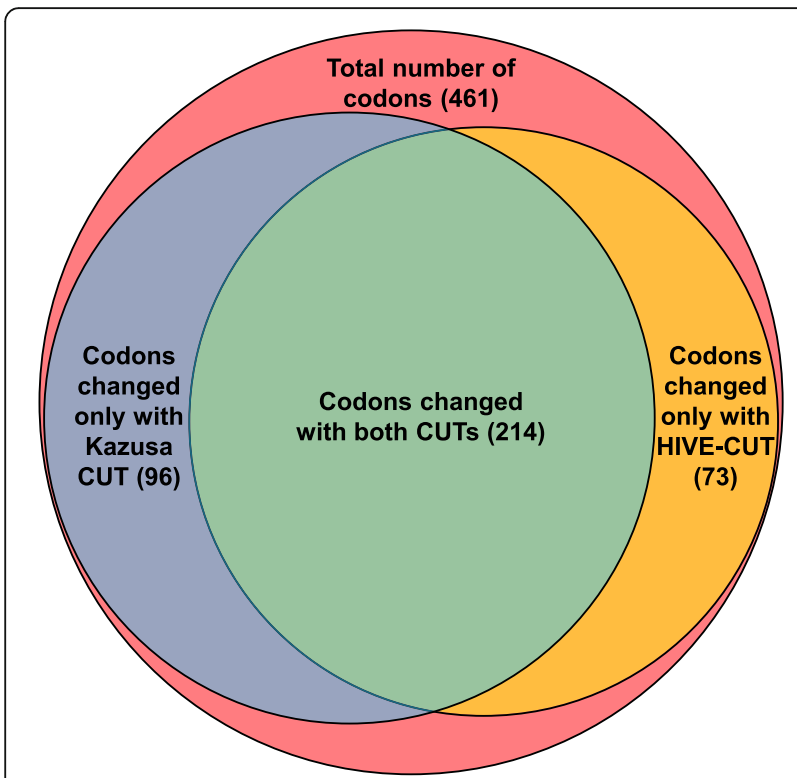

Fig. 4 Differences in codon optimization based on the HIVE-CUT and the Kazusa codon usage tables. The HIVE-CUT and the Kazusa codon usage tables were entered in the codon optimization algorithm ATGme to determine the number of suboptimal codons [39]. The Venn diagram shows how many codons were determined to be sub-optimal in the human coagulation factor IX gene for expression in $\mathrm{CHO}$ (Cricetulus griseus) cells. The codon usage tables used appear in Additional file 2

proteins. Homo sapiens and Cricetulus griseus (Chinese hamster) are not very different in terms of their codon usage bias. Furthermore, in 2007, Cricetulus griseus had not been extensively sequenced, and therefore its codon usage tables were not accurate. Using human coagulation factor IX, a gene that is commonly codon optimized for clinical applications like gene therapy [56-58], as an example, it is clear that accurate codon usage data is critical for optimization strategies. As illustrated in Fig. 4, when using codon usage tables that were last updated in 2007 to optimize the human coagulation factor IX gene for expression in $\mathrm{CHO}$ cells using ATGme [39], 310 codons are identified as suboptimal. Performing the same analysis with HIVE-CUTs resulted in 287 optimized codons, only 214 of which were the same as when using the older tables. Performing codon optimization or harmonization based on inaccurate codon usage tables would, therefore, be ineffective. Alternatively, when the tRNA supplementation approach is used, codon usage, of a single gene or the entire genome, can be compared to tRNA levels to determine which tRNA may require supplementation. Availability of tRNA can be either estimated computationally $[10,59]$ or measured experimentally.

An area of research that has been recently gaining attention is whether synonymous codon substitutions have effects on protein translation beyond levels of expression [32, 60-62]. Codon frequency has coevolved to correlate with tRNA concentration [63-66]. As a result, translational efficiency at the codon level is affected by tRNA abundance. It is generally accepted that rare codons are translated slower than the common ones and it has been shown that rare codons often cluster [40]. These rare codon clusters can induce pauses during translation that have experimentally been shown to affect cotranslational folding [20]. Although there is little consensus regarding patterns of rare codon clusters and secondary protein structure, there is data from an array of yeast species suggesting that coil regions of a protein

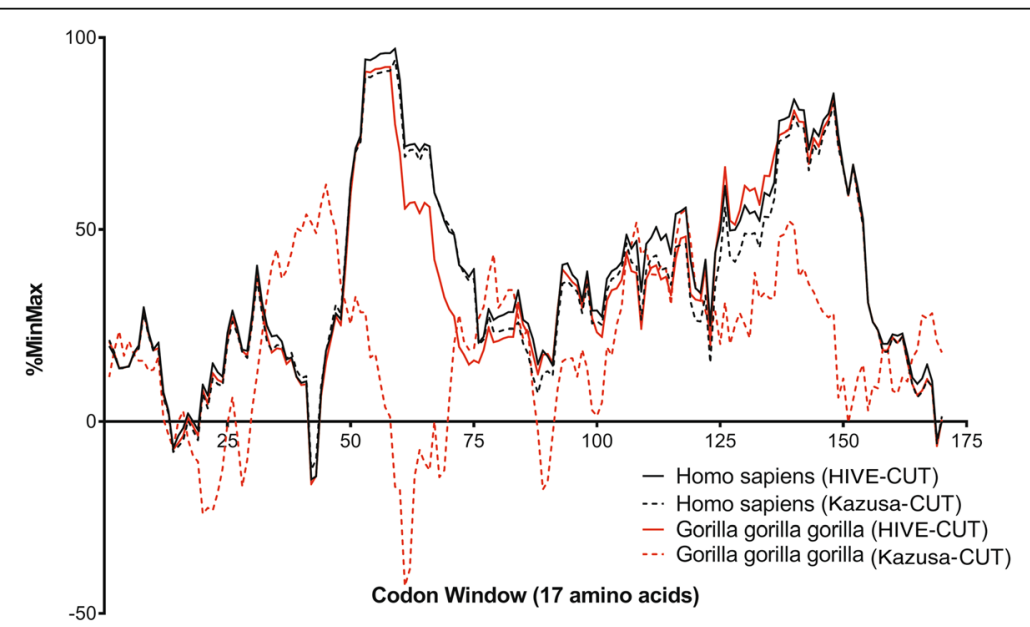

Fig. 5 Rare codon cluster distribution based on the HIVE-CUT and the Kazusa codon usage tables. The \%MinMax algorithm [40] was implemented to generate results for the interferon beta-1b gene sequence of Homo sapiens and Gorilla gorilla gorilla. The human and gorilla proteins have similar amino acid sequences and show similar results with the HIVE-CUT; however, highly divergent results were observed with Kazusa CUTs. The codon usage tables for these species used in the calculation of the translation rate appear in Additional file 3 
are depleted of common codons while $\beta$-sheets are depleted of rare codons [67]. Interestingly, coil regions are comprised of loops that fold before exit of the ribosomal tunnel. In contrast, $\beta$-sheet domains are topologically discontinuous and must await synthesis to begin folding [67]. Collectively this data supports a causal relationship between codon choice, translation rate and protein structure.

The availability of codon usage tables that span a very wide range of species can be instrumental in unravelling the role of codon choice on co-translational folding. Algorithms that evaluate the relative rareness of codons in a nucleotide sequence used to produce a given protein sequence [40] can serve as a rough proxy for the local translation rate, and the presence of translational pauses due to rare codons can be studied. To obtain a more accurate estimation of rare codon clusters, accurate codon usage tables are required. For example, when examining the rare codon distribution of the human interferon beta- $1 \mathrm{~b}$ a number of potential translational pauses are apparent. A similar pattern of rare codon clusters is also observed with the gorilla sequence of interferon beta-1b, which is due both to the similarity of the amino acid sequence in the two species but also due to similarities in codon usage bias (Fig. 5). If, however, an older codon usage table had been used, a dramatically different pattern of rare codon clusters would have been generated giving rise to false conclusions (Fig. 5). Comparing the rare codon distribution of a human protein to those of closely related species has proven useful in determining the functional role of synonymous mutations [67] and how they may cause disease [32].

\section{Conclusions}

Codon usage bias plays a role in many biological processes, and substitution of synonymous codons is a very common technique in industry and research. Accurate codon usage data is an important part of many common bioinformatics tools that incorporate the effects of codon usage bias into their analyses. This database is a dramatic improvement over existing databases. It is more comprehensive in terms of the number of species included and more accurate due to the vastly larger sources and improved quality of sequencing data and their associated annotations.

\section{Availability and requirements}

Project name: HIVE-Codon Usage Tables

Project home page: https://hive.biochemistry.gwu.edu/ review/codon

Operating system: Platform independent

Programming languages: Python 2.7 , Javascript, $\mathrm{C}++$ Other requirements: Web browser (Chrome or Firefox) License: The database is publicly available

\section{Additional file}

Additional file 1: HIVE-CUT screenshot showing search results for Homo sapiens using the RefSeq and GenBank databases. (DOCX $154 \mathrm{~kb}$ )

Additional file 2: Homo sapiens and Cricetulus griseus CUTs. This table contains the data used to create Fig. 4 of the main text. (DOCX 22 kb)

Additional file 3: Homo sapiens and Gorilla gorilla gorilla CUTs. This table contains the data used to create Fig. 5 of the main text. (DOCX 24 kb)

\section{Abbreviations}

BCT: Bacterial (GenBank division); CDS: Coding DNA sequence; CGI: Common gateway interface; CHO: Chinese hamster ovary; CUT: Codon usage table; DDD: Data-driven documents; DNA: Deoxyribonucleic acid; DOM: Document object model; ENV: Environmental (GenBank division); EST: Expressed sequence tag (GenBank division); GC: Guanine-cytosine; HIVE: Highperformance integrated virtual environment; HTML: Hypertext markup language; INV: invertebrate (GenBank division); MAM: Other mammalian (GenBank division); NCBI: National center for biotechnology information; PHG: Phage (GenBank division); PLN: Plant and fungal (GenBank division); PRI: Primate (GenBank division); RNA: Ribonucleic acid; ROD: Rodent (GenBank division); SQL: Structured query language; taxID: Taxonomy ID number; tRNA: Transfer RNA; VRL: Viral (GenBank division); VRT: Other vertebrate (GenBank division); XML: Extensible markup language

\section{Acknowledgements}

We thank Nobuko Katagiri and Ryan Hunt from DPPT/OTAT/CBER/FDA, and Paul Kitts and Avi Kimchi from NIH/NLM/NCBI, for fruitful discussions. This work was funded by internal grants from the Hemostasis Branch/Division of Plasma Protein Therapeutics/Office of Tissues and Advanced Therapies/ Center for Biologics Evaluation and Research of the U.S. Food and Drug Administration. Software development, hardware and maintenance of public portal are supported by the HIVE project at FDA and at George Washington University. This research was supported in part by an appointment to the Research Participation Program at the Center for Biologics Evaluation and Research administered by the Oak Ridge Institute for Science and Education through an interagency agreement between the U.S. Department of Energy and the U.S. Food and Drug Administration.

\section{Funding}

This work was supported by funds from the Hemostasis Branch, Center for Biologics Evaluation and Research, FDA (CK-S). The FDA operating funds were used here to support the stipend of the computational fellow. The biologist involved in this project is a Full Time Employee of the federal government. The research is aligned with the FDA mission.

Availability of data and materials

The datasets analysed during the current study are available in NCBI's FTP repository, ftp://ftp.ncbi.nlm.nih.gov/.

All data generated during this study are available in the HIVE-CUT webpage, hive.biochemistry.gwu.edu/review/codon. This database is available for use by all, including both academics and non-academics.

\section{Authors' contributions}

AA, JA, and CK-S conceived the project. JA developed the HIVE-CUTS software. $J A, E O, A R$, and LV S-Q developed the web interface. AA wrote the manuscript. JA and UK generated figures and assisted in the writing of the manuscript. VS supervised the software and web interface development. CK-S supervised the project. All authors tested and suggested modifications in the website, and reviewed and approved the manuscript.

Ethics approval and consent to participate

Not applicable.

Consent for publication

Not applicable.

Competing interests

The authors declare that they have no competing interests. 


\section{Publisher's Note}

Springer Nature remains neutral with regard to jurisdictional claims in published maps and institutional affiliations.

\section{Author details Administration, Silver Spring, USA. ${ }^{2}$ High Performance Integrated Administration, Silver Spring, USA. \\ Received: 19 June 2017 Accepted: 15 August 2017 Published online: 02 September 2017}

'Division of Plasma Protein Therapeutics, Office of Tissue and Advanced Therapies, Center for Biologics Evaluation and Research, Food and Drug Environment, Center for Biologics Evaluation and Research, Food and Drug

\section{References}

1. Grantham R, Gautier C, Gouy M, Mercier R, Pave A. Codon catalog usage and the genome hypothesis. Nucleic Acids Res. 1980;8(1):r49-62.

2. dos Reis M, Savva R, Wernisch L. Solving the riddle of codon usage preferences: a test for translational selection. Nucleic Acids Res. 2004;32(17):5036-44.

3. Sharp PM, Averof M, Lloyd AT, Matassi G, Peden JF. DNA sequence evolution: the sounds of silence. Philos Trans R Soc Lond Ser B Biol Sci. 1995:349(1329):241-7.

4. Duret L. Evolution of synonymous codon usage in metazoans. Curr Opin Genet Dev. 2002;12(6):640-9.

5. Plotkin JB, Kudla G. Synonymous but not the same: the causes and consequences of codon bias. Nat Rev Genet. 2011;12(1):32-42.

6. Novoa EM, Ribas de Pouplana L. Speeding with control: codon usage, tRNAs, and ribosomes. Trends Genet. 2012;28(11):574-81.

7. Sharp PM, Stenico M, Peden JF, Lloyd AT. Codon usage: mutational bias, translational selection, or both? Biochem Soc Trans. 1993;21(4):835-41.

8. Sharp PM, Li WH. The codon adaptation index-a measure of directional synonymous codon usage bias, and its potential applications. Nucleic Acids Res. 1987:15(3):1281-95.

9. Wright $F$. The 'effective number of codons' used in a gene. Gene. 1990;87(1):23-9.

10. Sabi $R$, Tuller T. Modelling the efficiency of codon-tRNA interactions based on codon usage bias. DNA Res. 2014;21(5):511-26.

11. Benson DA, Cavanaugh M, Clark K, Karsch-Mizrachi I, Lipman DJ, Ostell J, Sayers EW. GenBank. Nucleic Acids Res. 2013;41(Database issue):D36-42.

12. Pruitt K, Brown G, Tatusova T, et al. The reference sequence (RefSeq) database. In: McEntyre J, Ostell J, editors. The NCBI handbook (internet). Bethesda, MD: National Center for Biotechnology Information (US); 2002.

13. Wong EH, Smith DK, Rabadan R, Peiris M, Poon LL. Codon usage bias and the evolution of influenza a viruses. Codon usage biases of influenza virus. BMC Evol Biol. 2010;10:253.

14. Anhlan D, Grundmann N, Makalowski W, Ludwig S, Scholtissek C. Origin of the 1918 pandemic H1N1 influenza a virus as studied by codon usage patterns and phylogenetic analysis. RNA. 2011;17(1):64-73.

15. Prat $Y$, Fromer $M$, Linial $N$, Linial $M$. Codon usage is associated with the evolutionary age of genes in metazoan genomes. BMC Evol Biol. 2009;9:285.

16. Bahir I, Fromer M, Prat Y, Linial M. Viral adaptation to host: a proteome-based analysis of codon usage and amino acid preferences. Mol Syst Biol. 2009;5:311.

17. Pandit A, Sinha S. Differential trends in the codon usage patterns in HIV-1 genes. PLoS One. 2011;6(12):e28889.

18. Shin YC, Bischof GF, Lauer WA, Desrosiers RC. Importance of codon usage for the temporal regulation of viral gene expression. Proc Natl Acad Sci U S A. 2015;112(45):14030-5.

19. Anfinsen CB. Principles that govern the folding of protein chains. Science. 1973;181(4096):223-30.

20. Sander IM, Chaney JL, Clark PL. Expanding Anfinsen's principle: contributions of synonymous codon selection to rational protein design. J Am Chem Soc. 2014;136(3):858-61.

21. Buhr F, Jha S, Thommen M, Mittelstaet J, Kutz F, Schwalbe H, Rodnina MV, Komar AA. Synonymous Codons direct Cotranslational folding toward different protein conformations. Mol Cell. 2016;61(3):341-51.

22. O'Brien EP, Ciryam P, Vendruscolo M, Dobson CM. Understanding the influence of codon translation rates on cotranslational protein folding Acc Chem Res. 2014;47(5):1536-44.

23. Angov E. Codon usage: nature's roadmap to expression and folding of proteins. Biotechnol J. 2011;6(6):650-9.
24. Bali V, Bebok Z. Decoding mechanisms by which silent codon changes influence protein biogenesis and function. Int J Biochem Cell Biol. 2015;64:58-74.

25. Weatheritt RJ, Babu MM. Evolution. The hidden codes that shape protein evolution. Science. 2013;342(6164):1325-6.

26. Warnecke T, Weber CC, Hurst LD. Why there is more to protein evolution than protein function: splicing, nucleosomes and dual-coding sequence. Biochem Soc Trans. 2009;37(Pt 4):756-61.

27. Chamary JV, Parmley JL, Hurst LD. Hearing silence: non-neutral evolution at synonymous sites in mammals. Nat Rev Genet. 2006;7(2):98-108.

28. Tuller T, Zur H. Multiple roles of the coding sequence $5^{\prime}$ end in gene expression regulation. Nucleic Acids Res. 2015;43(1):13-28.

29. Hilterbrand A, Saelens J, Putonti C. CBDB: the codon bias database. BMC Bioinf. 2012;13:62

30. CUB - DB Codon Usage Bias Database. http://cub-db.cs.umt.edu/index.shtml. Accessed 2 June 2017.

31. Nakamura Y, Gojobori T, Ikemura T. Codon usage tabulated from international DNA sequence databases: status for the year 2000. Nucleic Acids Res. 2000;28(1):292

32. Pizzo L, Iriarte A, Alvarez-Valin F, Marin M. Conservation of CFTR codon frequency through primates suggests synonymous mutations could have a functional effect. Mutat Res. 2015;775:19-25.

33. Gerdol M, De Moro G, Venier P, Pallavicini A. Analysis of synonymous codon usage patterns in sixty-four different bivalve species. PeerJ. 2015;3:e1520.

34. Cock PJA, Antao T, Chang JT, Chapman BA, Cox CJ, Dalke A, Friedberg I, Hamelryck T, Kauff F, Wilczynski B, et al. Biopython: freely available python tools for computational molecular biology and bioinformatics. Bioinformatics. 2009;25(11):1422-3.

35. Sayers EW, Barrett T, Benson DA, Bryant SH, Canese K, Chetvernin V Church DM, DiCuccio M, Edgar R, Federhen S, et al. Database resources of the National Center for biotechnology information. Nucleic Acids Res. 2009;37(Database issue):D5-15.

36. Simonyan V, Chumakov K, Dingerdissen H, Faison W, Goldweber S, Golikov A, Gulzar N, Karagiannis K, Vinh Nguyen Lam P, Maudru T, et al. High-performance integrated virtual environment (HIVE): a robust infrastructure for next-generation sequence data analysis. Database (Oxford). 2016;2016

37. Bostock M, Ogievetsky V, Heer J. D(3): Data-Driven Documents. IEEE Trans Vis Comput Graph. 2011:17(12):2301-9.

38. HIVE-Codon Usage Table Database. https://hive.biochemistry.gwu.edu/ review/codon. Accessed 16 June 2017.

39. Daniel E, Onwukwe GU, Wierenga RK, Quaggin SE, Vainio SJ, Krause M. ATGme: open-source web application for rare codon identification and custom DNA sequence optimization. BMC Bioinf. 2015;16:303.

40. Clarke TF, Clark PL. Rare codons cluster. PLoS One. 2008;3(10):e3412

41. Williams DP, Regier D, Akiyoshi D, Genbauffe F, Murphy JR. Design, synthesis and expression of a human interleukin-2 gene incorporating the codon usage bias found in highly expressed Escherichia Coli genes. Nucleic Acids Res. 1988;16(22):10453-67.

42. Kotula L, Curtis PJ. Evaluation of foreign gene codon optimization in yeast: expression of a mouse IG kappa chain. Biotechnology (N Y). 1991;9(12):1386-9.

43. Perlak FJ, Fuchs RL, Dean DA, McPherson SL, Fischhoff DA. Modification of the coding sequence enhances plant expression of insect control protein genes. Proc Natl Acad Sci U S A. 1991;88(8):3324-8.

44. Zolotukhin S, Potter M, Hauswirth WW, Guy J, Muzyczka N. A "humanized" green fluorescent protein cDNA adapted for high-level expression in mammalian cells. J Virol. 1996;70(7):4646-54.

45. Hale RS, Thompson G. Codon optimization of the gene encoding a domain from human type 1 neurofibromin protein results in a threefold improvement in expression level in Escherichia Coli. Protein Expr Purif. 1998;12(2):185-8.

46. Zhou Z, Schnake $P$, Xiao L, Lal AA. Enhanced expression of a recombinant malaria candidate vaccine in Escherichia Coli by codon optimization. Protein Expr Purif. 2004:34(1):87-94

47. Gustafsson C, Govindarajan S, Minshull J. Codon bias and heterologous protein expression. Trends Biotechnol. 2004;22(7):346-53.

48. Angov E, Hillier CJ, Kincaid RL, Lyon JA. Heterologous protein expression is enhanced by harmonizing the Codon usage frequencies of the target gene with those of the expression host. PLoS One. 2008;3(5):e2189.

49. Angov E, Legler PM, Mease RM. Adjustment of Codon usage frequencies by Codon harmonization improves protein expression and folding. In: JTC E, 
Xu M-Q, editors. Heterologous gene expression in Ecoli: methods and protocols. Totowa, NJ: Humana Press; 2011. p. 1-13.

50. Brinkmann U, Mattes RE, Buckel P. High-level expression of recombinant genes in Escherichia Coli is dependent on the availability of the dnaY gene product. Gene. 1989;85(1):109-14.

51. Baca AM, Hol WG. Overcoming codon bias: a method for high-level overexpression of plasmodium and other AT-rich parasite genes in Escherichia Coli. Int J Parasitol. 2000;30(2):113-8.

52. Kane JF. Effects of rare codon clusters on high-level expression of heterologous proteins in Escherichia Coli. Curr Opin Biotechnol. 1995;6(5):494-500.

53. Ou KC, Wang CY, Liu KT, Chen YL, Chen YC, Lai MD, Yen MC. Optimization protein productivity of human interleukin-2 through codon usage, gene copy number and intracellular tRNA concentration in $\mathrm{CHO}$ cells. Biochem Biophys Res Commun. 2014;454(2):347-52

54. Li A, Kato Z, Ohnishi H, Hashimoto K, Matsukuma E, Omoya K, Yamamoto Y, Kondo N. Optimized gene synthesis and high expression of human interleukin-18. Protein Expr Purif. 2003;32(1):110-8.

55. Maertens B, Spriestersbach A, von Groll U, Roth U, Kubicek J, Gerrits M, Graf M, Liss M, Daubert D, Wagner R, et al. Gene optimization mechanisms: a multi-gene study reveals a high success rate of full-length human proteins expressed in Escherichia Coli. Protein Sci. 2010;19(7):1312-26.

56. Cantore A, Nair N, Della Valle P, Di Matteo M, Màtrai J, Sanvito F, Brombin C, Di Serio C, Angelo A, Chuah M, et al. Hyperfunctional coagulation factor IX improves the efficacy of gene therapy in hemophilic mice. Blood. 2012:120(23):4517.

57. Nathwani AC, Reiss UM, Tuddenham EGD, Rosales C, Chowdary P, Mclntosh J, Della Peruta M, Lheriteau E, Patel N, Raj D, et al. Long-term safety and efficacy of factor IX gene therapy in hemophilia B. N Engl J Med. 2014;371 (21):1994-2004.

58. Zhang R, Wang Q, Zhang L, Chen S. Optimized human factor IX expression cassettes for hepatic-directed gene therapy of hemophilia B. Front Med. 2015;9(1):90-9.

59. Chan PP, Lowe TM. GtRNAdb: a database of transfer RNA genes detected in genomic sequence. Nucleic Acids Res. 2009;37(Database issue):D93-7.

60. Kimchi-Sarfaty C, Oh JM, Kim IW, Sauna ZE, Calcagno AM, Ambudkar SV, Gottesman MM. A "silent" polymorphism in the MDR1 gene changes substrate specificity. Science. 2007;315(5811):525-8

61. Cortazzo P, Cervenansky C, Marin M, Reiss C, Ehrlich R, Deana A. Silent mutations affect in vivo protein folding in Escherichia Coli. Biochem Biophys Res Commun. 2002;293(1):537-41.

62. Parmley JL, Hurst LD. How do synonymous mutations affect fitness? BioEssays. 2007;29(6):515-9.

63. Ikemura T. Correlation between the abundance of Escherichia Coli transfer RNAs and the occurrence of the respective codons in its protein genes. J Mol Biol. 1981;146(1):1-21.

64. Akashi H. Synonymous codon usage in Drosophila Melanogaster: natural selection and translational accuracy. Genetics. 1994;136(3):927-35.

65. Moriyama EN, Powell JR. Codon usage bias and tRNA abundance in drosophila. J Mol Evol. 1997;45(5):514-23.

66. Duret L. tRNA gene number and codon usage in the C. Elegans genome are co-adapted for optimal translation of highly expressed genes. Trends Genet. 2000;16(7):287-9.

67. Pechmann S, Frydman J. Evolutionary conservation of codon optimality reveals hidden signatures of cotranslational folding. Nat Struct Mol Biol. 2013;20(2):237-43

\section{Submit your next manuscript to BioMed Central and we will help you at every step:}

- We accept pre-submission inquiries

- Our selector tool helps you to find the most relevant journal

- We provide round the clock customer support

- Convenient online submission

- Thorough peer review

- Inclusion in PubMed and all major indexing services

- Maximum visibility for your research

Submit your manuscript at www.biomedcentral.com/submit

CBiomed Central 